\title{
Diversity at deep-sea hydrothermal vent and intertidal mussel beds
}

\author{
Cindy Lee Van Dover ${ }^{1, *}$, Jennifer L. Trask ${ }^{2}$ \\ 'Biology Department, College of William \& Mary, Williamsburg, Virginia 23187, USA \\ ${ }^{2}$ Institute of Marine Science, University of Alaska Fairbanks, Fairbanks, Alaska 99775, USA
}

\begin{abstract}
Hydrothermal vents are well-known for their exotic faunas, but common ecological descriptors of communities, apart from species lists, have eluded vent ecologists for decades. Using replicate sampling, we examine species richness, diversity, evenness, and composition within mussel beds at the Lucky Strike hydrothermal vent field on the Mid-Atlantic Ridge, and we compare these measures to those found at intertidal mussel beds along the south-central Alaskan coast using similar sampling efforts. The number of species found at the vent mussel beds is less than half that of the intertidal mussel beds. From calculations of diversity and evenness indices, we find that the lower diversity of the vent mussel beds is a reflection of a reduced number of rare species found there compared to intertidal mussel beds. In contrast to relatively even distributions of deep-sea soft-sediment (non-vent) communities, both vent and intertidal mussel beds share the attribute of very uneven distributions, with a large number of individuals found in a small number of species. Based on comparisons of the Shannon diversity index $(H)$ within vent mussel beds and in a variety of other marine environments, we find that diversity at vent mussel beds is low, but no lower than the diversity measured in certain shallowwater environments. We hypothesize that diversity within the Lucky Strike mussel beds, located on a slow-spreading ridge where the spacing between vents is large, represents the low extreme of diversity at hydrothermal vents. We expect higher diversity at mussel beds on fast-spreading ridges (e.g., on the East Pacific Rise).
\end{abstract}

KEY WORDS: Diversity $\cdot$ Hydrothermal vent $\cdot$ Mussel beds $\cdot$ Lucky Strike $\cdot$ Alaska

\section{INTRODUCTION}

Hydrothermal vents in the deep sea are often cited as examples of ecosystems with high productivity but low diversity (Wright et al. 1993), yet for more than a decade, quantitative measures of diversity at vents were limited to the work of Grassle and his colleagues, based on a small number of box core samples in soft sediments (Grassle et al. 1985, Dinet et al. 1988, Petrecca \& Grassle 1990, Grassle \& Petrecca 1994). Lower diversity at soft-sediment vent sites compared to the non-vent deep sea was also observed in an analysis of nematodes by Vanreusal et al. (1997). But soft sediments are the exception rather than the rule for hydrothermal systems. They are found only along mid-

\footnotetext{
•E-mail: cindy_vandover@wm.edu
}

ocean ridge spreading centers where sedimentation rates are high, such as Guaymas Basin in the Gulf of California and Escanaba Trough on the Gorda Ridge (Van Dover 2000). Measures of diversity or species richness from the much more typical, hard substratum (basalt or sulfide mineral) vent communities have usually been restricted to lists of species (e.g., Tunnicliffe 1988, Tunnicliffe et al. 1998) and data sets that are difficult to compare from one vent field or geographic region to the next due to differences in sampling methods, effort, and presence or absence of specific microhabitats (Underwood \& Petraitis 1993).

We present here the first quantitative measure of species richness for a typical vent habitat and we provide a comparison to a shallow-water, intertidal analogue. We specifically examine diversity in mussel beds, which are widely distributed at deep-sea hydrothermal vents on the East Pacific Rise, Mid-Atlantic 
Ridge, and western Pacific back-arc basins, as well as at a variety of deep-sea cold seeps and in the shallow intertidal environment. In intertidal regions, mussel beds have become a standard unit for assessment of regional and global diversity patterns because they are so widely distributed and because they offer complex secondary surfaces and interstitial habitats for a large number of associated species that can be sampled as discrete units (Lohse 1993, Seed 1996). Mussel beds at deep-sea vents also support a variety of invertebrates and are thus a suitable structural habitat for biodiversity comparisons at hydrothermal vents within and between ocean basins and between ecosystems driven by photosynthetic and chemosynthetic production. Restricted by the sampling opportunities available to us, we began our study of invertebrate diversity in vent mussel beds at the mussel-dominated Lucky Strike hydrothermal system on the Mid-Atlantic Ridge and we compare this system with an equal sampling effort at an intertidal mussel bed on the south-central coast of Alaska. This work is now part of a larger biodiversity program that will compare vent mussel bed diversity within and between ocean basins as well as at individual vent mussel beds over time.

\section{METHODS}

Vent mussel beds are located at the Lucky Strike hydrothermal field at $37^{\circ} 17.5^{\prime} \mathrm{N}, 32^{\circ} 16^{\prime} \mathrm{W}$ on the MidAtlantic Ridge $(1600 \mathrm{~m})$. Descriptions of the setting can be found in Van Dover et al. (1996), Langmuir et al. (1997), Comtet \& Desbruyères (1998) and Von Damm et al. (1998). Biomass of the vent fauna at Lucky Strike is dominated exclusively by an undescribed species of vent mussel in the genus Bathymodiolus. These mussels host chemoautotrophic and methanotrophic endosymbiotic bacteria (Trask \& Van Dover 1999) and occupy low-temperature $\left(5\right.$ to $\left.10^{\circ} \mathrm{C}\right)$, diffuse flow zones on surfaces of large sulfide structures. Two sulfide structures, Eiffel Tower and Sintra (located approximately $400 \mathrm{~m}$ apart), were chosen as sampling sites. In July 1996, we collected 5 replicate, $\sim 2$ I volumes of mussels from each site using the unmanned, remotely operated vehicle (ROV) 'Jason'. Mussel clumps were harvested by Jason's manipulator and placed in individual sample boxes with lids to prevent washing during ascent. There was inevitably some loss of individuals during transfer from the mussel bed to the sample boxes, making our samples best described as 'semi-quantitative'.

Intertidal mussel Mytilus trossulus beds are located on the south-central coast of Alaska, near the University of Alaska's Kasitsna Bay Marine Laboratory in Seldovia (Kenai Peninsula). We selected 2 discontin- uous mussel zones (Eagle Island and Jakolof Point) occurring at the same tidal height and separated by approximately $400 \mathrm{~m}$. Five replicate, 2 l volumes of mussels were collected from each site at low tide. Because we anticipated a seasonal effect on diversity at this intertidal site, this sampling program was repeated at 3 mo intervals throughout 1 yr (September 1996 to June 1997).

Once collected, vent and intertidal samples were treated identically. Mussel clumps were wrapped in clear plastic and sample volume was measured by displacement. Mussels were then unwrapped and a new displacement volume was obtained. Interstitial volume of the clump was determined by difference between the 2 volumes. All samples were washed 3 times in filtered seawater and these washings were passed through a $63 \mu \mathrm{m}$ sieve to retain the associated fauna. This associated fauna was fixed in buffered $10 \%$ seawater formalin and stored in $70 \% \mathrm{EtOH}$. Mussel sizefrequency distributions were calculated using mussel lengths measured with digital calipers. Sieved samples were sorted twice under a dissecting microscope (the second time after staining with Rose Bengal) and individuals were identified to the lowest taxonomic category possible and enumerated.

Unidentifiable larval stages were included in the subsequent analyses only when they clearly belonged to taxa not already represented by adult stages. Species commensal in mussels were not included (i.e., the polynoid polychaete Branchipolynoe cf. seepensis, which lives in the mantle cavity of the vent mussel). We also arbitrarily chose not to include mussels $>5 \mathrm{~mm}$ in our diversity analyses. Mussels $<5 \mathrm{~mm}$ represent individuals that are scaled to the same size as the rest of the mussel-associated fauna and were deemed to be interactive rather than structural at this stage of their life history.

Species identifications of the vent fauna were made with the assistance of specialists and are reliable to morphological species. Exceptions are the vent amphipod samples, which are overwhelmingly dominated by females of a single species (cf. Bouvieriella curtirama Bellan-Santini \& Thurston), but from which we may yet resolve an additional rare or singleton amphipod species. We estimate that the error in our measure of species richness introduced by the absence of complete knowledge of the amphipod taxa is $<7 \%$ (i.e., maximum total number of species in the vent mussel bed might be 30 instead of 28). Taxonomy of some of the Alaskan fauna is less well-known than the vent fauna and was more problematic. We were assisted by local specialists at the Institute of Marine Science (University of Alaska Fairbanks), but where taxa are designated as 'spp.', we did not have the expertise to make species-level identifications based on morphology. The 
scope of this study did not include completion of a definitive species list for Kasitsna Bay. Our measures of diversity in the intertidal mussel bed should thus be interpreted as a minimum estimate.

Diversity and evenness indices were calculated from combined data from all 10 replicates within a system using formulas presented in Hayek \& Buzas (1997). To maintain similar sample sizes for comparisons between vent and intertidal mussel beds, we use only the September data for the intertidal calculations.

\section{RESULTS}

Mussels at the vent site were larger than mussels collected from the intertidal site (overall mean lengths $=48$ vs $30 \mathrm{~mm}$, respectively) and there was a correspondingly larger mean number of mussels in a given volume at the intertidal site (Table 1 ; size-frequency data are given in Fig. 1). Interstitial volumes (i.e., habitat space) were similar between vent and intertidal samples (Table 1). Mussel size-frequency distributions provide important information about the relative ages and habitat quality of mussel bed patches when making comparisons within an ecosystem (Tsuchiya \& Nishihira 1986), but it is outside the goals of this study to attempt to interpret the effect of mussel size frequencies on diversity in comparisons between vent and intertidal sites. We infer from visual observations and interstitial volume data that mussel beds at both sites provide qualitatively similar spatial habitats with complex interstitial volumes.

Species lists and abundances for each replicate sample from vent and intertidal (September) samples are provided in Tables $2 \& 3$. These tables also give a record of individual sample volumes so that the data can be normalized. Abundances of dominant species among replicates are highly variable in both the vent and intertidal samples, indicating that both habitats are heterogeneous with respect to the distribution of associated species.

Per volume of habitat, the intertidal site had a higher overall average abundance of individuals than the vent

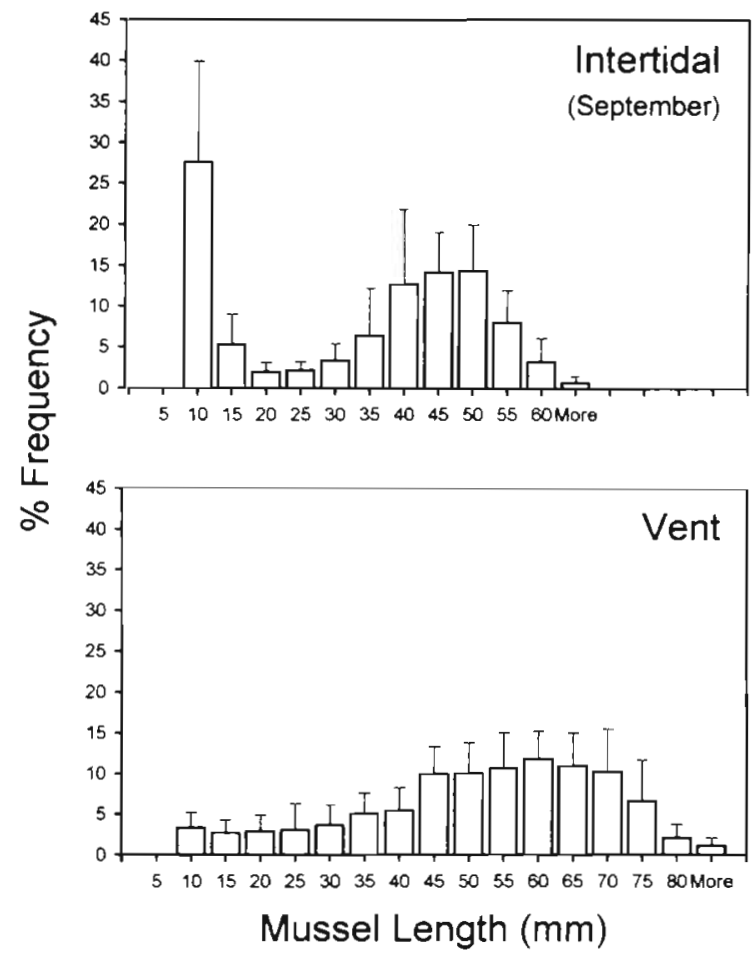

Fig. 1. Mussel size frequency distributions (mean and standard deviations for each size class from 5 replicate samples at each of 2 locations within a site) for intertidal Mytilus trossulus (September sample only) and deep-sea vent Bathymodiolus sp.

site (1724 vs 816 ind. $1^{-1}$ mussel volume). Biomass, while not quantified, was also patently higher at the intertidal site than at the vent site. Seasonal data (September, December, March, June) for the intertidal sites are tabulated as summary data only (i.e., combining counts from 5 replicates at each of 2 sites; Table 4); comprehensive worksheets for these sampling periods are available from the authors.

Seasonal intertidal data and vent data are summarized graphically as species-effort curves (Fig. 2), using mussel volume as our best measure of sampling effort. Species richness $(S)$ is lower within Lucky Strike mussel beds $(S=28)$ than during any sampling period within the intertidal mussel beds (overall $S=76$ ).

Table 1 Comparison of vent and intertidal (September sample only) mussel bed structural characteristics. Values are means (n replicates) \pm standard deviation

\begin{tabular}{|c|c|c|c|c|c|c|}
\hline & \multicolumn{3}{|c|}{ Vent } & \multicolumn{3}{|c|}{ Intertidal (September) } \\
\hline & $\begin{array}{c}\text { Eiffel Tower } \\
(n=5)\end{array}$ & $\begin{array}{l}\text { Sintra } \\
(n=5)\end{array}$ & $\begin{array}{l}\text { Overall } \\
(\mathrm{n}=10)\end{array}$ & $\begin{array}{l}\text { Eagle Island } \\
\qquad(n=5)\end{array}$ & $\begin{array}{l}\text { Jakalof Point } \\
\qquad(\mathrm{n}=5)\end{array}$ & $\begin{array}{l}\text { Overall } \\
(n=10)\end{array}$ \\
\hline No. of mussels $>5$ mm length & $133 \pm 70$ & $167 \pm 37$ & $150 \pm 70$ & $217 \pm 69$ & $184 \pm 27$ & $200 \pm 52$ \\
\hline Length (mm) & $49 \pm 16$ & $48 \pm 19$ & $48 \pm 18$ & $27 \pm 3$ & $34 \pm 1$ & $30 \pm 4$ \\
\hline Mussel volume (l) & $2.4 \pm 0.5$ & $2.2 \pm 0.6$ & $2.3 \pm 0.5$ & $2.6 \pm 0.4$ & $2.4 \pm 0.4$ & $2.5 \pm 0.4$ \\
\hline Interstitial volume (l) & $1.0 \pm 0.2$ & $1.1 \pm 0.3$ & $1.1 \pm 0.3$ & $0.9 \pm 0.2$ & $0.9 \pm 0.2$ & $0.9 \pm 0.2$ \\
\hline
\end{tabular}


Table 2. Vent mussel bed. Species-abundance data by replicate

\begin{tabular}{|c|c|c|c|c|c|c|c|c|c|c|c|}
\hline \multirow[t]{2}{*}{ Phylum } & \multirow[t]{2}{*}{ Species } & \multicolumn{5}{|c|}{ Eiffel Tower } & \multicolumn{5}{|c|}{ Sintra } \\
\hline & & 1 & 2 & 3 & 4 & 5 & 1 & 2 & 3 & 4 & 5 \\
\hline Nemertea & Nemertea sp. & 0 & 1. & 0 & 2 & 7 & 61 & 0 & 0 & 0 & 1 \\
\hline Nematoda & Nematoda sp. & 40 & 64 & 0 & 0 & 1. & 52 & 3 & 8 & 230 & 118 \\
\hline \multirow[t]{6}{*}{ Annelida } & Levensteiniella sp. & 24 & 1 & 5 & 0 & 3 & 4 & 1 & 1 & 17 & 2 \\
\hline & Opisthotrochopodus sp. & 85 & 0 & 6 & 0 & 7 & 21 & 5 & 28 & 83 & 29 \\
\hline & Harmothoinae sp. & 0 & 0 & 0 & 0 & 0 & 1 & 0 & 0 & 0 & 0 \\
\hline & Amathys lutzi & 1 & 3 & 3 & 0 & 0 & 5 & 11 & 0 & 8 & 2 \\
\hline & Dorvilleidae sp. & 0 & 0 & 0 & 0 & 0 & 2 & 0 & 0 & 3 & 0 \\
\hline & Unidentified sack-like worm & 0 & 1 & 0 & 0 & 0 & 1 & 0 & 0 & 0 & 0 \\
\hline \multirow[t]{9}{*}{ Mollusca } & Lepetodrilus sp. & 3 & 1 & 4 & 1 & 0 & 8 & 7 & 4 & 2 & 4 \\
\hline & Pseudorimula midatlantica & 0 & 0 & 6 & 0 & 0 & 0 & 0 & 0 & 1 & 1 \\
\hline & Paralepetopsis sp. & 0 & 0 & 0 & 0 & 1 & 0 & 0 & 0 & 0 & 0 \\
\hline & Shinkailepas sp. & 0 & 0 & 0 & 0 & 0 & 0 & 4 & 1 & 0 & 0 \\
\hline & Protolira valvatoides & 0 & 0 & 0 & 1 & 1 & 49 & 2 & 0 & 0 & 2 \\
\hline & Laeviphitus sp. & 0 & 0 & 0 & 0 & 0 & 1 & 1 & 0 & 0 & 0 \\
\hline & Orbitestillidae sp. & 0 & 0 & 1 & 0 & 0 & 0 & 0 & 0 & 0 & 0 \\
\hline & Peltospira sp. & 0 & 0 & 0 & 0 & 0 & 0 & 1 & 0 & 0 & 0 \\
\hline & Bathymodiolus sp. $(<5 \mathrm{~mm})$ & 27 & 53 & 115 & 35 & 51 & 425 & 146 & 167 & 107 & 229 \\
\hline \multirow[t]{10}{*}{ Arthropoda } & Ostracoda sp. 1 & 1558 & 194 & 14 & 8 & 107 & 1130 & 58 & 413 & 3608 & 229 \\
\hline & Ostracoda sp. 2 & 0 & 0 & 0 & 0 & 0 & 0 & 0 & 0 & 1 & 0 \\
\hline & Aphotopontius temperatus & 36 & 24 & 11 & 8 & 23 & 164 & 6 & 38 & 149 & 137 \\
\hline & Aphotopontius atlanteus & 654 & 152 & 358 & 97 & 228 & 192 & 82 & 130 & 140 & 359 \\
\hline & Stygiopontius rimivagus & 0 & 0 & 0 & 0 & 0 & 7 & 0 & 0 & 0 & 0 \\
\hline & Copepoda sp. 1 & 3 & 2 & 2 & 1 & 7 & 200 & 5 & 13 & 66 & 6 \\
\hline & Copepoda sp. 2 & 0 & 0 & 0 & 1 & 0 & 1 & 0 & 0 & 0 & 0 \\
\hline & Copepoda sp. 3 & 0 & 0 & 0 & 0 & 0 & 1 & 0 & 1 & 0 & 0 \\
\hline & Chorocaris fortunata & 2 & 1 & 0 & 2 & 5 & 1.1 & 27 & 4 & 4 & 8 \\
\hline & A.mphipoda spp. & 303 & 267 & 710 & 1495 & 609 & 535 & 314 & 174 & 765 & 1770 \\
\hline Echinodermata & Ophiuroida sp. & 1 & 0 & 0 & 0 & 0 & 0 & 0 & 0 & 0 & 0 \\
\hline \multicolumn{2}{|c|}{ Total number of individuals } & 2737 & 764 & 1235 & 1651 & 1050 & 2871 & 673 & 982 & 5184 & 2897 \\
\hline \multicolumn{2}{|c|}{ Total number of species } & 13 & 13 & 12 & 11 & 13 & 21 & 14 & 13 & 15 & 15 \\
\hline \multicolumn{2}{|c|}{ Sample volume (1) } & 2.2 & 2.6 & 3 & 2.1 & 3 & 2.3 & 2 & 2.4 & 2.1 & 3 \\
\hline
\end{tabular}

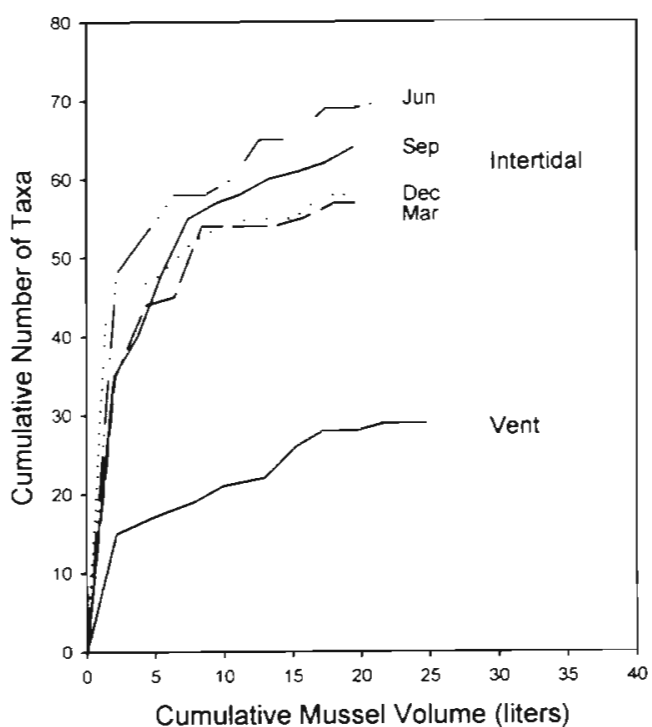

Fig. 2. Species-effort curves for invertebrate fauna associated with intertidal and deep-sea vent mussel beds. Each curve is made up of 10 points (i.e., from 5 replicate samples at each of 2 locations within a site). Cumulative sample volume is expressed in liters of mussels, determined by displacement
Using a variety of indices ( $S$, Fisher's $\alpha$, Simpson's $\lambda_{\text {, }}$ Shannon's $H$ ) we find that diversity is higher within the intertidal mussel bed than in the vent mussel bed by a factor of 1.2 to 2.6 (Table 5). Two measures of evenness (Pielou's $J$ and Buzas \& Gibson's $E$ ) are also reported in Table 5 . Similarities in $J$ and $E$ between vent and intertidal samples indicate that individuals are partitioned among species in a similar manner. This is illustrated further by examination of relative abundance and species rank order for both vent and intertidal samples (Fig. 3). In both cases, a small number of species $(\leq 5)$ account for a large percentage $(90 \%)$ of the total abundance of individuals.

Proportional distribution of species among major taxonomic groups (arthropods, gastropods, polychaetes, other) is similar between vent and intertidal mussel beds (Fig. 4). The specific nature of the most abundant taxa, however, is distinct between the 2 sites (Fig. 4), with small motile crustaceans (amphipods, ostracods and copepods) making up $>90 \%$ of the individuals collected from the Lucky Strike vent mussel beds (Table 6), in contrast to nematodes, copepods, mites, and several small polychaete species dominating the intertidal mussel 
Table 3. Intertidal mussel bed. Species-abundance data by replicate for the September sampling period

\begin{tabular}{|c|c|c|c|c|c|c|c|c|c|c|c|}
\hline \multirow[t]{2}{*}{ Phylum } & \multirow[t]{2}{*}{ Species } & \multicolumn{5}{|c|}{ Eagle Island } & \multicolumn{5}{|c|}{ Jakolof Point } \\
\hline & & 1 & 2 & 3 & 4 & 5 & 1 & 2 & 3 & 4 & 5 \\
\hline \multirow[t]{2}{*}{ Sarcodina } & Foraminiferā sp. 1 & 39 & 1 & 35 & 100 & 0 & 8 & 31 & 5 & 9 & 51 \\
\hline & Foraminifera sp. 2 & 7 & 4 & 4 & 22 & 1 & 9 & 7 & 2 & 5 & 11 \\
\hline Cnidaria & Actiniidae sp. & 0 & 0 & 0 & 5 & 2 & 0 & 0 & 0 & 0 & 0 \\
\hline \multirow{3}{*}{ Platyhelminthes } & Kaburakia excelsa & 0 & 0 & 0 & 1 & 0 & 0 & 2 & 0 & 0 & 1 \\
\hline & Turbellaria sp. 1 & 0 & 1 & 0 & 1 & 0 & 0 & 0 & 0 & 25 & 7 \\
\hline & Turbellaria sp. 2 & 0 & 0 & 0 & 0 & 0 & 0 & 0 & 0 & 0 & 1 \\
\hline Nemertea & Nemertea sp. & 6 & 7 & 8 & 18 & 5 & 85 & 47 & 18 & 21 & 20 \\
\hline \multirow[t]{2}{*}{ Nematoda } & Nematoda sp. 1 & 592 & 531 & 430 & 755 & 1819 & 1315 & 1923 & 733 & 382 & 2050 \\
\hline & Nematoda sp. 2 & 9 & 1 & 0 & 116 & 8 & 32 & 29 & 12 & 7 & 88 \\
\hline \multirow{14}{*}{ Annelida } & Oligochaeta spp. & 29 & 26 & 10 & 6 & 3 & 0 & 0 & 2 & 1 & 5 \\
\hline & Typosyllis $\mathrm{sp}$ & 2 & 4 & 9 & 16 & 3 & 8 & 15 & 6 & 2 & 8 \\
\hline & Sphaerosyllis sp. & 0 & 0 & 0 & 0 & 5 & 0 & 1 & 0 & 0 & 3 \\
\hline & Brania sp. & 0 & 0 & 0 & 1 & 0 & 0 & 0 & 0 & 0 & 0 \\
\hline & Exogone sp. & 14 & 10 & 2 & 8 & 18 & 6 & 4 & 8 & 12 & 2 \\
\hline & Nerilla sp. & 136 & 143 & 354 & 116 & 0 & 76 & 714 & 31 & 0 & 327 \\
\hline & Nereis sp. & 0 & 3 & 3 & 0 & 1 & 1 & 2 & 2 & 3 & 3 \\
\hline & Pholoe minuta & 37 & 3 & 37 & 27 & 0 & 3 & 1 & 2 & 1 & 3 \\
\hline & Sabellidae sp. & 46 & 6 & 11 & 94 & 5 & 181 & 363 & 259 & 7 & 221 \\
\hline & Naineris sp. & 0 & 0 & 1 & 2 & 0 & 0 & 1 & 0 & 0 & 7 \\
\hline & Terebellidae sp. & 0 & 0 & 0 & 1 & 0 & 0 & 0 & 0 & 0 & 0 \\
\hline & Phyllodocidae sp. & 0 & 0 & 0 & 0 & 0 & 1 & 10 & 2 & 1 & 3 \\
\hline & Eunicidae sp. & 0 & 0 & 0 & 0 & 0 & 0 & 0 & 0 & 0 & 3 \\
\hline & Ampharetidae sp. & 0 & 0 & 0 & 0 & 0 & 0 & 1 & 0 & 0 & 0 \\
\hline \multirow[t]{18}{*}{ Mollusca } & Margarites beringensis & 36 & 16 & 27 & 52 & 0 & 64 & 77 & 23 & 26 & 156 \\
\hline & Littorina sitkana & 18 & 6 & 9 & 1 & 5 & 6 & 1 & 11 & 9 & 5 \\
\hline & Cingula sp. & 16 & 2 & 10 & 22 & 1 & 16 & 13 & 19 & 3 & 67 \\
\hline & Alvinia sp. & 4 & 1 & 1 & 18 & 0 & 1 & 24 & 0 & 0 & 37 \\
\hline & Nucella lamellosa & 1 & 0 & 0 & 0 & 0 & 0 & 0 & 0 & 0 & 0 \\
\hline & Nucella lima & 2 & 2 & 0 & 0 & 0 & 1 & 0 & 0 & 1 & 0 \\
\hline & Odostomia sp. & 0 & 0 & 0 & 3 & 1 & 0 & 0 & 0 & 0 & 4 \\
\hline & Buccinum baen & 0 & 4 & 0 & 1 & 0 & 2 & 0 & 0 & 1 & 0 \\
\hline & Moelleria sp. & 0 & 0 & 0 & 0 & 0 & 0 & 0 & 1 & 0 & 0 \\
\hline & Siphonaria sp. & 0 & 1 & 0 & 0 & 0 & 0 & 0 & 1 & 0 & 0 \\
\hline & Hiatella arctica & 1 & 2 & 1. & 8 & 1 & 0 & 1 & 0 & 0 & 1 \\
\hline & Onchidella borealis & 0 & 0 & 2 & 2 & 1 & 0 & 2 & 0 & 0 & 0 \\
\hline & Lottia pelta & 2 & 6 & 3 & 8 & 4 & 8 & 0 & 0 & 2 & 5 \\
\hline & Lottia digitalis & 0 & 0 & 0 & 0 & 0 & 0 & 0 & 0 & 1 & 0 \\
\hline & Tectura scutum & 0 & 0 & 1 & 0 & 3 & 0 & 0 & 0 & 0 & 1 \\
\hline & Protothaca staminea & 0 & 0 & 1 & 0 & 0 & 0 & 1 & 0 & 0 & 0 \\
\hline & Mytilus trossulus $(<5 \mathrm{~mm})$ & 432 & 160 & 212 & 286 & 154 & 272 & 399 & 315 & 47 & 335 \\
\hline & Turtonia minuta & 0 & 0 & 1 & 0 & 1 & 26 & 19 & 9 & 1 & 7 \\
\hline Arthropoda & Pseudoscorpionida sp. & 0 & 0 & 1 & 0 & 0 & 0 & 1 & 0 & 0 & 0 \\
\hline & Acari sp. 1 & 273 & 97 & 255 & 248 & 110 & 213 & 522 & 322 & 167 & 327 \\
\hline & Acari sp. 2 & 15 & 14 & 1 & 11 & 5 & 7 & 8 & 9 & 4 & 11 \\
\hline & Insecta sp. 1 & 1 & 0 & 3 & 0 & 0 & 0 & 2 & 0 & 0 & 0 \\
\hline & Insecta sp. 2 & 0 & 0 & 2 & 0 & 0 & 0 & 0 & 0 & 0 & 0 \\
\hline & Insecta sp. 3 & 1 & 0 & 0 & 0 & 1 & 0 & 1 & 0 & 0 & 0 \\
\hline & Anurida maritima & 76 & 64 & 25 & 99 & 22 & 38 & 47 & 19 & 4 & 16 \\
\hline & Collembola sp. & 0 & 0 & 0 & 0 & 0 & 0 & 1 & 0 & 0 & 2 \\
\hline & Coleoptera sp. & 0 & 0 & 0 & 1 & 0 & 1 & 0 & 0 & 1 & 1 \\
\hline & Diptera spp. & 0 & 10 & 4 & 8 & 1 & 0 & 9 & 2 & 1 & 7 \\
\hline & Chironomidae spp. & 5 & 1 & 9 & 20 & 47 & 28 & 42 & 81 & 19 & 15 \\
\hline & Ostracoda sp. & 4 & 2 & 11 & 35 & 3 & 79 & 146 & 35 & 33 & 133 \\
\hline & Copepoda (Harpacticoida) spp. & 1069 & 552 & 457 & 684 & 1247 & 1152 & 16 & 619 & 516 & 1899 \\
\hline & Copepoda (Cyclopoida) spp. & 3 & 1 & 12 & 45 & 3 & 6 & 2 & 1 & 3 & 3 \\
\hline & Copepoda (Calanoida) spp. & 1 & 1 & 0 & 0 & 0 & 0 & 0 & 0 & 0 & 0 \\
\hline & Balanomorpha spp. & 30 & 23 & $?$ & 2 & 31 & 11 & 6 & 30 & 110 & 75 \\
\hline & Brachyura $\mathrm{sp}$ & 0 & 0 & 0 & 0 & 1 & 0 & 0 & 1 & 0 & 2 \\
\hline & Pagurus sp. & 1 & 8 & 10 & 7 & 19 & 0 & 3 & 0 & 7 & 0 \\
\hline & Amphipoda spp. & 6 & 10 & 7 & 7 & 9 & 0 & 3 & 2 & 4 & 0 \\
\hline & Munna ubiquita & 124 & 112 & 51 & 51 & 48 & 38 & 34 & 19 & 10 & 21 \\
\hline Echinodermata & Cucumaria vegae & 9 & 16 & 65 & 124 & 2 & 3 & 9 & 14 & 3 & 11 \\
\hline & Leptasterias hexactis & 0 & 0 & 2 & 1 & 4 & 0 & 1 & 1 & 0 & 0 \\
\hline Chordata & Anoplarchus purpurescens & 0 & 0 & 0 & 1 & 0 & 0 & 0 & 0 & 0 & 1 \\
\hline Total number of $\mathrm{i}$ & individuals & 3047 & 1851 & 2094 & 3034 & 3594 & 3697 & 4541 & 2616 & 1449 & 5956 \\
\hline Total number of $s$ & species & 35 & 37 & 40 & 43 & 36 & 32 & 43 & 34 & 36 & 44 \\
\hline Sample volume (l & & 2.0 & 1.7 & 1.8 & 1.9 & 2.1 & 1.6 & 2.1 & 4.4 & 1.8 & 2.1 \\
\hline
\end{tabular}


beds (Table 7 ). Mussel post-larvae and juveniles $(<5 \mathrm{~mm})$ were present in similarly large numbers ( 7 to $8 \%$ of individuals) in both vent and intertidal sites, consistent with active recruitment and comparable population dynamics of mussels within the 2 habitats.

\section{DISCUSSION}

Although we describe our sampling method as semi-quantitative, our measure of species richness $(S$ ) derived from the species effort curve shown in Fig. 2 is robust for the vent mussel bed samples, given that the curve reaches an asymptote. We suggest that despite a potential sampling bias against highly motile species, the 24.7 I cumulative sample volume allows us to estimate species richness within the mussel beds with confidence. And, while we highlight the potential bias error for the reader, the sampling method we used was in fact extremely effective in collecting large numbers of highly motile amphipods, ostracods, and copepods. Any incremental increase in sample volume is likely to pick up merely 1 additional singleton species (species represented by a single individual).

It is difficult and sometimes even counter-productive to make comparisons of species richness at hydrothermal vents using extant species lists generated from what have mostly been haphazard samples and uneven (and unquantified) sampling efforts. For example, in comparing species richness at the Lucky Strike mussel bed reported here (28 species) to the species list for the entire Mid-Atlantic Ridge as of 1996 (34 species; Tunnicliffe \& Fowler 1996), one might conclude that the Lucky Strike mussel beds are a relative hot spot of diversity on this ridge system, when in fact all we can state at present is that they are the most carefully studied, with a specific focus on a quantitative measure of diversity and sampling to an asymptote on a species-effort curve. A more complete sampling effort and species list for the $13^{\circ} \mathrm{N}$ vent fields on the East Pacific Rise yields a species richness of 100 taxa (Tunnicliffe \& Fowler 1996), but this list includes invertebrates associated with tubeworm, mussel, and alvinel-
Table 4. Intertidal mussel bed. Seasonal species-abundance lists. Seasonal sample data represent combined numbers of individuals from 5 replicates at each of 2 sites

\begin{tabular}{|c|c|c|c|c|c|}
\hline Phylum & Species & Sep & Dec & Mar & Jun \\
\hline \multirow[t]{2}{*}{ Sarcodina } & Foraminifera sp. 1 & 279 & 172 & 111 & 223 \\
\hline & Foraminifera sp. 2 & 72 & 53 & 82 & 101 \\
\hline Cnidaria & Actiniidae sp. & 7 & 0 & 9 & 6 \\
\hline \multirow[t]{3}{*}{ Platyhelminthes } & Kaburakia excelsa & 4 & 1 & 1 & 1 \\
\hline & Turbellaria sp. 1 & 34 & 84 & 58 & 109 \\
\hline & Turbellaria sp. 2 & 1 & 3 & 0 & 1 \\
\hline Nemertea & Nemertea sp. & 235 & 77 & 21 & 131 \\
\hline \multirow[t]{2}{*}{ Nematoda } & Nematoda sp. 1 & 10530 & 1632 & 1304 & 10413 \\
\hline & Nematoda sp. 2 & 302 & 88 & 29 & 37 \\
\hline \multirow[t]{18}{*}{ Annelida } & Oligochaeta spp. & 82 & 61 & 20 & 30 \\
\hline & Typosyllis sp. & 73 & 69 & 94 & 89 \\
\hline & Sphaerosyllis sp. & 9 & 7 & 8 & 1.2 \\
\hline & Brania sp. & 1 & 21 & 3 & 6 \\
\hline & Exogone sp. & 84 & 30 & 0 & 21 \\
\hline & Syllis sp. & 0 & 0 & 0 & 1 \\
\hline & Nerilla sp. & 1897 & 342 & 336 & 1681 \\
\hline & Nereis sp. & 18 & 10 & 0 & 10 \\
\hline & Harmothoe sp. & 0 & 0 & 0 & 5 \\
\hline & Pholoe minuta & 114 & 32 & 27 & 129 \\
\hline & Sabellidae sp. & 1193 & 259 & 117 & 949 \\
\hline & Naineris sp. & 11 & 0 & 0 & 9 \\
\hline & Terebellidae sp. & 1 & 1 & 1 & 2 \\
\hline & Phyllodocidae sp. & 17 & 6 & 6 & 17 \\
\hline & Eunicidae sp. & 3 & 1 & 1 & 0 \\
\hline & Ampharetidae sp. & 1 & 0 & 0 & 1 \\
\hline & Spionidae sp. & 0 & 5 & 1 & 4 \\
\hline & Maldanidae sp. & 0 & 0 & 1 & 0 \\
\hline \multirow[t]{21}{*}{ Mollusca } & Margarites beringensis & 477 & 282 & 61 & 192 \\
\hline & Littorina sitkana & 71 & 112 & 57 & 224 \\
\hline & Cingula sp. & 169 & 87 & 22 & 213 \\
\hline & Alvinia sp. & 86 & 59 & 19 & 86 \\
\hline & Lacuna sp. & 0 & 23 & 2 & 3 \\
\hline & Nucella lamellosa & 1 & 0 & 0 & 0 \\
\hline & Nucella lima & 6 & 6 & 11 & 27 \\
\hline & Odostomia sp. & 8 & 17 & 11 & 40 \\
\hline & Buccinum baeri & 8 & 12 & 7 & 50 \\
\hline & Moelleria sp. & 1 & 0 & 0 & 0 \\
\hline & Siphonaria sp. & 2 & 1 & 1 & 4 \\
\hline & Hiatella arctica & 15 & 15 & 19 & 19 \\
\hline & Onchidella borealis & 7 & 7 & 8 & 36 \\
\hline & Lottia pelta & 38 & 31 & 15 & 22 \\
\hline & Lottia digitalis & 1 & 7 & 6 & 5 \\
\hline & Tectura scutum & 5 & 12 & 5 & 21 \\
\hline & Katharina tunicata & 0 & 0 & 0 & 2 \\
\hline & Tonicella sp. & 0 & 0 & 0 & 9 \\
\hline & Protothaca staminea & 2 & 0 & 1 & 9 \\
\hline & Mytilus trossulus (<5 mm) & 2612 & 1696 & 905 & 740 \\
\hline & Turtonia minuta & 64 & 13 & 3 & 48 \\
\hline
\end{tabular}

lid structural habitats, each of which supports a variety of invertebrate taxa. There is, to our knowledge, no published breakdown of species richness within these distinctive habitats nor is there any quantitative assessment of the degree of species overlap between habitats. Thus, while the Lucky Strike mussel beds indeed have less than $1 / 3$ the species richness of the total vent community at $13^{\circ} \mathrm{N}$ on the East Pacific Rise, it is impossible to know at this point whether species 
Table 4 (continued)

\begin{tabular}{|c|c|c|c|c|c|}
\hline Phylum & Species & Sep & Dec & Mar & Jun \\
\hline \multirow[t]{24}{*}{ Arthropoda } & Pseudoscorpionida sp. & 2 & 0 & 1 & 2 \\
\hline & Acari sp. 1 & 2534 & 802 & 573 & 2943 \\
\hline & Acari sp. 2 & 85 & 38 & 10 & 32 \\
\hline & Acari sp. 3 & 0 & 2 & 0 & 0 \\
\hline & Insecta sp. 1 & 6 & 0 & 0 & 0 \\
\hline & Insecta sp. 2 & 2 & 0 & 0 & 0 \\
\hline & Insecta sp. 3 & 3 & 0 & 0 & 0 \\
\hline & Insecta sp. 4 & 0 & 0 & 0 & 1 \\
\hline & Anurida maritima & 410 & 124 & 71 & 1392 \\
\hline & Collembola sp. & 3 & 1 & 0 & 8 \\
\hline & Coleoptera sp. & 4 & 0 & 0 & 0 \\
\hline & Diptera spp. & 42 & 6 & 6 & 11 \\
\hline & Chironomidae spp. & 267 & 519 & 13 & 9 \\
\hline & Nebalia sp. & 0 & 0 & 0 & 1 \\
\hline & Ostracoda sp. & 481 & 275 & 139 & 44 \\
\hline & Copepoda (Harpacticoida) spp. & 8211 & 3003 & 2212 & 8053 \\
\hline & Copepoda (Cyclopoida) spp. & 79 & 77 & 25 & 30 \\
\hline & Copepoda (Calanoida) spp. & 2 & 1 & 0 & 1 \\
\hline & Balanomorpha spp. & 325 & 193 & 150 & 140 \\
\hline & Brachyura sp. & 4 & 0 & 0 & 0 \\
\hline & Pagurus sp. & 55 & 6 & 1 & 15 \\
\hline & Amphipoda spp. & 48 & 15 & 10 & 62 \\
\hline & Munna ubiquita & 508 & 128 & 40 & 57 \\
\hline & Munna chromatocephala & 0 & 0 & 0 & 2 \\
\hline \multirow[t]{3}{*}{ Echinodermata } & Cucumaria vegae & 256 & 81 & 167 & 454 \\
\hline & Leptasterias hexactis & 9 & 5 & 1 & 64 \\
\hline & Strongylocentrotus droebachiensis & 0 & 0 & 1 & 1 \\
\hline Chordata & Anoplarchus purpurescens & 2 & 0 & 0 & 1 \\
\hline \multicolumn{2}{|c|}{ Total number of individuals } & 31879 & 10610 & 6803 & 29061 \\
\hline \multicolumn{2}{|c|}{ Total number of species } & 64 & 54 & 53 & 66 \\
\hline \multicolumn{2}{|c|}{ Sample volume (l) } & 19.4 & 19.4 & 20.2 & 21.7 \\
\hline
\end{tabular}

however, by the lumping of 3 ridge systems and multiple habitats into a single northeast Pacific species list, and by the absence of any kind of mussel bed as a structural habitat at northeast Pacific vents. A further confounding factor in all of these comparisons is the temporal scale of sampling. Were we to sample Lucky Strike over as many years or on as many occasions as $13^{\circ} \mathrm{N}$ or northeast Pacific vents have been sampled, we would expect to expand the Lucky Strike species list if only because vents pass through hydrothermal cycles during which species lists can change significantly in response to waxing and waning of hydrothermal flow (Van Dover pers. obs.).

Because the June and September sampling efforts for the intertidal site do not reach an asymptote, ours is an underestimate of the maximum seasonal species richness in this system and of the difference in diversity between intertidal and vent mussel bed assemblages. It is clear from these species-effort curves that species richness at the intertidal mussel bed is more than twice that of the vent mussel bed, even during seasons when diversity is lowest in the intertidal beds.

richness in the $13^{\circ} \mathrm{N}$ mussel beds is higher than that of Lucky Strike mussel beds. A total of 74 species are reported from hydrothermal vents on the northeast Pacific ridge system (Gorda, Juan de Fuca, and Explorer ridges; Tunnicliffe \& Fowler 1996), making vents of this ridge system more diverse than the Lucky Strike mussel beds. The comparison is confounded,

Table 5. Comparison of diversity and evenness indices at vent and intertidal mussel beds. The 'enrichment factor' is the ratio of intertidal:vent indices. $S$ : species richness

\begin{tabular}{|lccc|}
\hline Index & Vent & Intertidal $^{\text {a }}$ & Enrichment factor \\
\hline Diversity & & & \\
$S$ & 28 & 64 & 2.2 \\
Fisher's $\alpha$ & 2.4 & 6.2 & 2.6 \\
Simpson's $\lambda^{b}$ & 0.27 & 0.19 & 1.4 \\
Shannon's H & 1.60 & 2.16 & 1.2 \\
Evenness & & & - \\
Pielou's J & 0.47 & 0.51 & - \\
Buzas \& Gibson's E & 0.17 & 0.14 & \\
a September sample only & & \\
'Decreases with increasing diversity \\
\hline
\end{tabular}

Diversity indices (Fisher's $\alpha$, Simpson's $\lambda$, and Shannon's $H$ ) also demonstrate that diversity is higher in the intertidal mussel bed than at the vent, but the degree of enrichment is index-dependent, ranging from a factor of 1.2 (Shannon's $H$ ) to 2.6 (Fisher's $\alpha$ ). Each diversity index provides different information. Fisher's $\alpha$ is a number close to the number of species expected to be represented by only a single (i.e., rare) individual (Hayek \& Buzas 1997). The higher value of $\alpha$ at the intertidal site than at the vent mussel bed is consistent with a larger number of rare species in the intertidal bed. Simpson's $\lambda$ is the probability that 2 individuals chosen at random from a sample belong to the same species (Hayek \& Buzas 1997). The slightly higher $\lambda$ value at the vent site thus indicates that individuals are slightly more concentrated in fewer species than at the intertidal site. Shannon's $H$ provides a measure of uncertainty in the identity of an individual pulled randomly from the sample (Hayek \& Buzas 1997), with low $H$ indicating a fairly high certainty of outcome (i.e., low diversity). $H$ is thus insensitive to rare (especially singleton) species and is not a good measure for discriminating this aspect of vent and intertidal samples. 


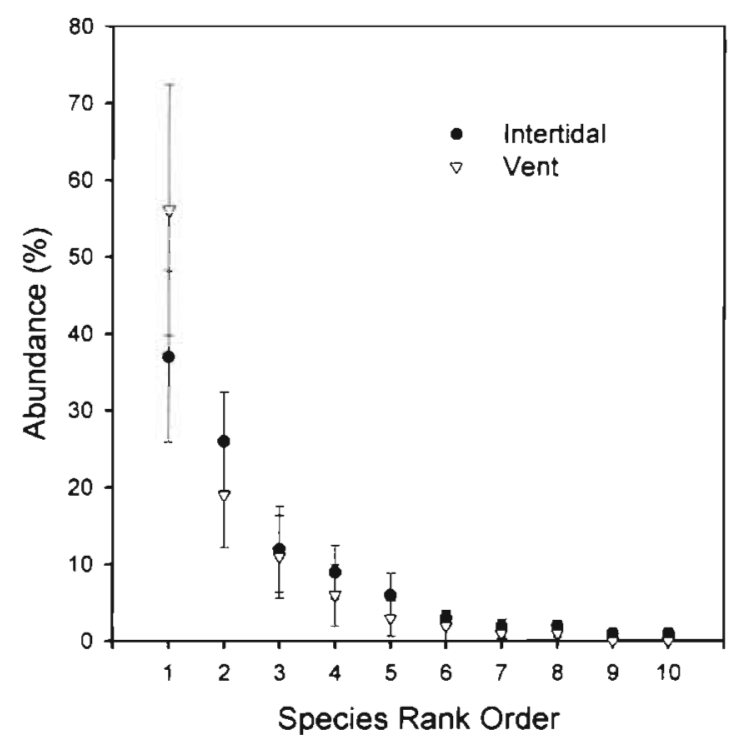

Fig. 3. Comparison of species rank order abundance for deepsea vent and intertidal mussel beds. Intertidal data are from September samples only. Error bars are standard deviations derived from 5 replicate samples at 2 locations within a site

$S$ and $H$ are common diversity measures reported for marine communities. $S$ values comparable to what we found in North Atlantic vent mussel beds (28 taxa) are reported for some Mytilus edulis beds in Japan (32 taxa; Tsuchiya \& Nishihira 1985, 1986), but $S$ values reported in other $M$. edulis beds in Japan (up to 69 taxa; Tsuchiya \& Nishihira 1985, 1986), as well as in $M$. edulis beds of North Wales (56 taxa; Lintas \& Seed 1994), Septifer virgatus beds of Hong Kong (52 taxa; Ong Che \& Morton 1992), and Brachidontes recurvus beds in Australia (56 species; Peake \& Quinn 1993) are more comparable to species richness in our September samples from Gulf of Alaska intertidal $M$. trossulus beds (64 species). Intermediate species richness is reported from $M$. galloprovincialis beds of southern France (99 taxa; Tsuchiya \& Bellan-Santini 1989). Highest mussel bed species richness is reported for $M$. californianus mussel beds of the Pacific coast of North

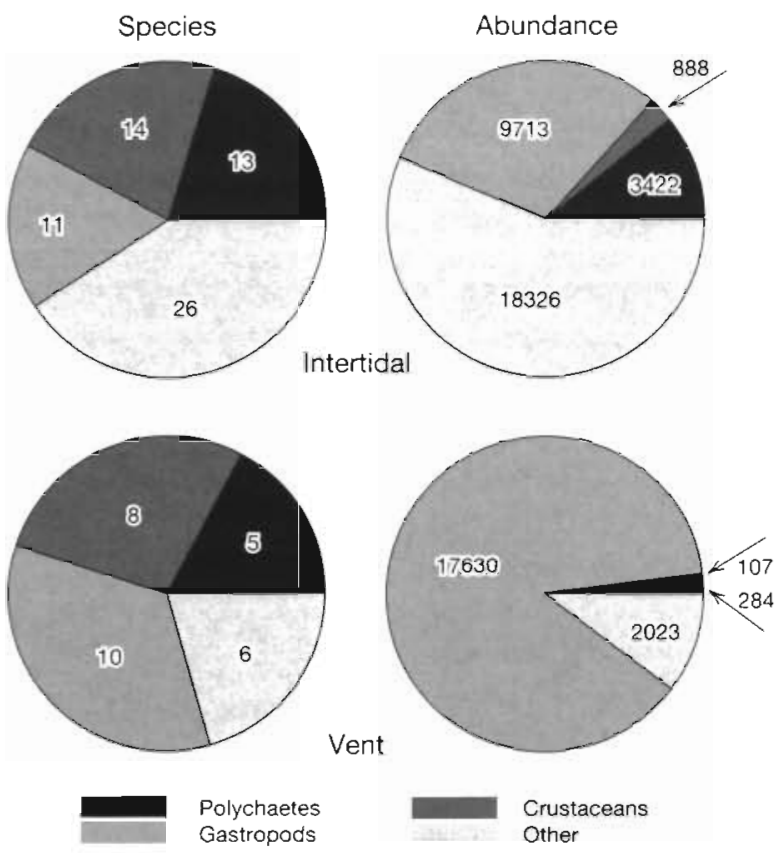

Fig. 4. Comparison of the distribution of species and abundance (numbers of individuals) for major taxa (polychaetes, gastropods, crustaceans, other) at vent and intertidal mussel beds

America (270 taxa; Suchanek 1979). While it is impossible to resolve fine-scale differences across ecosystems from comparisons of $H$ values due to variable sampling methods, biases, and effort among studies by different investigators, we can conclude that diversity at the Lucky Strike vent mussel bed $(H=1.6)$ is at the low end of the scale compared to North Atlantic $M$. edulis (North Wales) beds, where $H$ values range from 1.6 to 2.8 , depending on tidal height, patch size, and inclination of the substratum (lowest diversity in a high-shore pool and highest diversity in a low-shore, flat-rock mussel bed; Seed 1996). Lucky Strike vent mussel bed diversity is also at the low end of the range for values reported from other intertidal mussel beds and for typical coastal, estuarine, and deep-sea softsediment communities (Table 8 ).

Table 6. Abundance rankings of the 5 most common species in each sample; vent mussel bed. In 6 of 10 samples, for example, amphipods were most abundant (Species 1). Total $\mathrm{n}$ of the top 5 species for the vent mussel bed is 18886 (94\% of the total number of individuals)

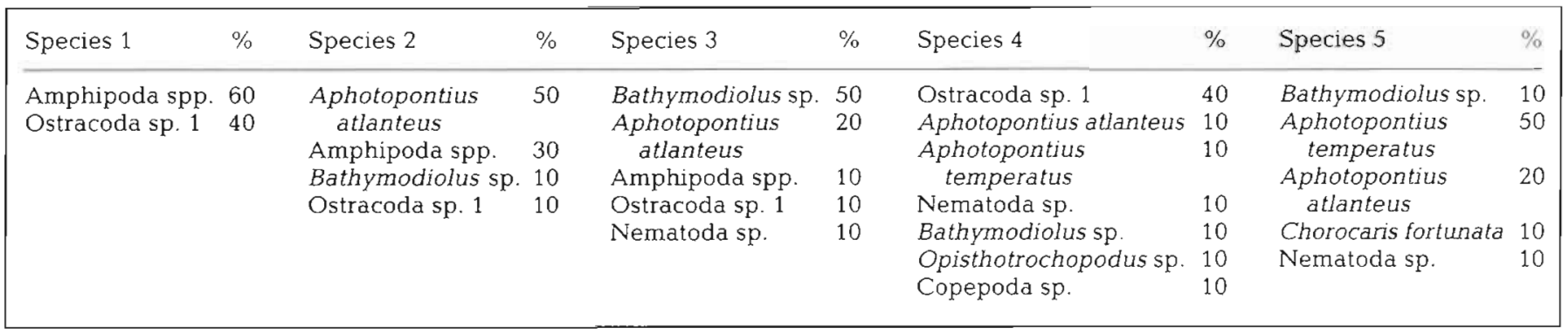


Table 7 Abundance rankings of the 5 most common species in each sample; intertidal mussel bed. In 6 of 10 samples, for example, nematodes were most abundant (Species 1). Total $\mathrm{n}$ of the top 5 species for the intertidal mussel bed is 27649 ( $86 \%$ of the total number of individuals)

\begin{tabular}{|c|c|c|c|c|c|c|c|c|c|}
\hline Species 1 & $\%$ & Species 2 & $\%$ & Species 3 & $\%$ & Species 4 & $\%$ & Species 5 & $\%$ \\
\hline Nematoda spp. & 60 & Copepods & 50 & Mytilus trossulus & 60 & Acari & 50 & Sabellidae & 30 \\
\hline \multirow{5}{*}{ Copepods } & 40 & Nematodes & 40 & Acari & 30 & Nerilla sp. & 20 & Munna ubiquita & 20 \\
\hline & & Nerilla sp. & 10 & Nerilla sp. & 10 & Mytilus trossulus & 20 & Mytilus trossulus & 20 \\
\hline & & & & & & Balanomorpha & 10 & Acari & 10 \\
\hline & & & & & & & & Cucumaria vegae & 10 \\
\hline & & & & & & & & Nerilla sp. & 10 \\
\hline
\end{tabular}

Table 8. Comparison of $H$ among selected marine environments

\begin{tabular}{|lcl|}
\hline Community & $H$ & Source \\
\hline Shallow soft sediment & $1.7-4.7$ & Steimle (1982), Ambrose (1993), Coleman et al. (1997) \\
Estuarine soft sediment & $1.6-4.9$ & Boesch (1973) \\
Deep-sea soft sediment & $5.7-6.0$ & Grassle \& Maciolek (1992), Vanreusel et al. (1997) \\
Intertidal mussel bed & $1.2-3.2$ & Seed (1996), Tsuchiya \& Nishihira (1985), Iwasaki (1995), this study \\
Deep-sed vent mussel bed & 1.6 & This study \\
\hline
\end{tabular}

Fauna associated with intertidal mussel beds have a high species richness but are typically dominated by a few very abundant species (Seed 1996). When we examine relative abundance data and species rank order in our samples, we find that the pattern of abundance is similar between vent and intertidal sites, with a small number of species or species groups accounting for a large percentage of the abundance. This contrasts with the much more even distribution of abundance among species that is characteristic of the non-vent deep-sea soft-sediment community (e.g., Grassle et al. 1985). Measures of evenness (Table 5) affirm this observation and lead us to conclude that low diversity at vent mussel beds is associated with a reduced number of rare species. This is consistent with the hypothesis that the chemical milieu of vent ecosystems imposes an adaptive filter on potential invaders. A lower number of rare species at vents may also be a consequence of the insular and ephemeral nature of vent habitats compared to that of intertidal mussel beds. A test of this latter hypothesis may be made by comparing diversity at vents along fast-versus slowspreading mid-ocean ridges. We expect, for example, that diversity should be highest at vents found on superfast-spreading ridges where vents are more closely spaced and less isolated from one another than on slowspreading ridges (Van Dover 2000). By this argument, diversity at Lucky Strike mussel beds, located on a slowspreading ridge, may represent the low-diversity extreme for vent mussel beds.

The putative functional character of the most abundant taxa at the vent and intertidal mussel beds is similar, namely, they are mostly small invertebrates that graze on the enriched particulate organic material associated with interstitial spaces of the mussel bed and that find refuge among the mussels from large but rare predators. Several explanations may account for higher abundances and biomass in the intertidal mussel beds, including combinations of lower primary productivity available to vent mussel bed fauna, higher predation rates within the vent mussel bed, or different rates of export of primary productivity from the local habitat. None of these possibilities has been investigated quantitatively. Note that while total primary production in vent mussel beds is likely to be comparable to or in excess of that available to an intertidal mussel bed, most of this vent production is locked in mussel/ endosymbiont biomass and may not be as readily accessed by the associated fauna as the primary production by phytoplankton and algae that fuels intertidal mussel bed assemblages.

For more than 2 decades, characterizations of fundamental descriptors of community structure at hardsubstratum hydrothermal vents have eluded ecologists. While it is clear that analysis of diversity in mussel bed assemblages may not represent total diversity at vents, recognition of mussel beds as credible and logistically feasible ecological units for comparison of species composition and diversity at vents allows us to move forward with global comparisons of mussel bed diversity, and with manipulative experiments that allow us to understand biotic and abiotic controls on species composition. Of particular interest is the degree to which fauna associated with mussel beds in different ocean basins are taxonomically and functionally 
convergent. Preliminary analysis of mussel bed fauna from the southern East Pacific Rise (Van Dover unpubl.) already reveals major differences in the dominant species, as well as higher biomass and higher diversity relative to the Lucky Strike vent samples.

Acknowledgements. We thank D. Fornari, S. Humphris, A Bowen and the ROV 'Jason' Deep-Submergence Operations Group, and the crew of the RV 'Knorr' for support at sea. Martin Sheader, Daniel Desbruyères, Arthur Humes, Marion Pettibone, Ann Vanreusel, and Anders Waren assisted with identifications of vent taxa. Stephanie Moreland from the Institute of Marine Science at the University of Alaska, Fairbanks, assisted with identifications of Alaska material. Jennifer Schurmeier helped with sorting samples. Ron Karlson, Fred Grassle and 2 anonymous individuals provided valuable critical reviews of the manuscript. This work was undertaken with funds from the $\mathrm{Na}$ tional Science Foundation and was first inspired by discussions with participants at the National Center for Ecological Analysis (NCEAS) workshop on deep-sea biodiversity.

\section{LITERATURE CITED}

Ambrose WG Jr (1993) Effects of predation and disturbance by ophiuroids on soft-bottom community structure in Oslofjord; results of a mesocosm study. Mar Ecol Prog Ser 97:225-236

Boesch DF (1973) Classification and community structure of macrobenthos in the Hampton Roads area, Virginia. Mar Biol 21:226-244

Coleman N, Gason AS, Poore GCB (1997) High species richness in the shallow marine waters of south-east Australia. Mar Ecol Prog Ser 154:17-26

Comtet T, Desbruyères D (1998) Population structure and recruitment in mytilid bivalves from the Lucky Strike and Menez Gwen hydrothermal vent fields $\left(37^{\circ} 17^{\prime} \mathrm{N}\right.$ and $37^{\circ} 50^{\prime} \mathrm{N}$ on the Mid-Atlantic Ridge). Mar Ecol Prog Ser 163:165-177

Dinet A, Grassle JF, Tunnicliffe V (1988) Premieres observations sur la meiofauna des sites hydrothermaux de la dorsale Est-Pacifique (Guaymas, $21^{\circ} \mathrm{N}$ ) et de $1^{\prime}$ Explorer Ridge. Oceanol Acta 85:7-14

Grassle JF, Maciolek N (1992) Deep-sea species richness: regional and local diversity estimates from quantitative bottom samples. Am Nat 139:313-341

Grassle JF, Petrecca R (1994) Soft-sediment hydrothermal vent communities of Escanaba Trough. USGS Bull 2002:327-335

Grassle JF, Brown-Leger LS, Morse-Porteous L, Petrecca R, Williams I (1985) Deep-sea fauna of sediments in the vicinity of hydrothermal vents. Biol Soc Wash Bull 6:443-452

Hayek LA, Buzas MA (1997) Surveying natural populations. Columbia University Press, New York

Iwasaki K (1995) Comparison of mussel bed community between two intertidal mytilids Septifer virgatus and Hormomya mutabilis. Mar Biol 123:109-119

Langmuir C, Humphris S, Fornari D, Van Dover C, Von Damm $\mathrm{K}$, Tivey $\mathrm{MK}$, Colodner $\mathrm{D}$, Charlou JL, Desonie $\mathrm{D}$, Wilson C, Fouquet Y, Klinkhammer G, Bougault $H$ (1997) Hydrothermal vents near a mantle hot spot: the Lucky Strike vent field at $37^{\circ} \mathrm{N}$ on the Mid-Atlantic Ridge. Earth Planet Sci Lett 148:69-91

Lintas C, Seed R (1994) Spatial variation in the fauna associated with Mytilus edulis on a wave-exposed rocky shore. J Molluscan Stud 60:165-174

Lohse DP (1993) The importance of secondary substratum in a rocky intertidal community. J Exp Mar Biol Ecol 166:1-17

Ong Che RG, Morton B (1992) structure and seasonal varia- tions in abundance of the macroinvertebrate community associated with Septifer virgatus (Bivalvia:Mytilidae) at Cape d'Aguilar, Hong Kong. Asian Mar Biol 9:217-233

Peake J, Quinn GP (1993) Temporal variation in species-area curves for invertebrates in clumps of an intertidal mussel. Ecography 16:269-277

Petrecca R, Grassle JF (1990) Notes on fauna from several deep-sea hydrothermal vent and cold seep soft-sediment communities. In: McMurray GR (ed) Gorda Ridge: a seafloor spreading center in the United States' Exclusive Economic Zone. Springer Verlag, New York, p 279-283

Seed R (1996) Patterns of biodiversity in the macro-invertebrate fauna associated with mussel patches on rocky shores. J Mar Biol Assoc UK 76:203-210

Steimle FW Jr (1982) The benthic macroinvertebrates of the Block Island Sound. Estuar Coast Shelf Sci 15:1-16

Suchanek TH (1979) The Mytilus californianus community: studies on the composition, structure, organization and dynamics of a mussel bed. PhD thesis, University of Washington, Seattle

Trask JL, Van Dover CL (1999) Site-specific and ontogenetic variations in nutrition of mussels (Bathymodiolus sp.) from the Lucky Strike hydrothermal vent field, Mid-Atlantic Ridge. Limnol Oceanogr 44:334-343

Tsuchiya M, Bellan-Santini D (1989) Vertical distribution of shallow rocky shore organisms and community structure of mussel beds (Mytilus galloprovincialis) along the coast of Marseille, France. Bull Mus His Nat Mars 49:91-110

Tsuchiya M, Nishihira M (1985) Islands of Mytilus edulis as a habitat for small intertidal animals; effect of island size on community structure. Mar Ecol Prog Ser 25:71-81

Tsuchiya M, Nishihira M (1986) Islands of Mytilus edulis as a habitat for small intertidal animals, effect of Mytilus age structure on the species composition of the associated fauna and community organization. Mar Ecol Prog Ser 31:171-178

Tunnicliffe V (1988) Biogeography and evolution of hydrothermal-vent fauna in the eastern Pacific Ocean. Proc R Soc Lond B 233:347-366

Tunnicliffe V, Fowler M (1996) Influence of sea-floor spreading on the global hydrothermal vent fauna. Nature 379: $531-533$

Tunnicliffe V, McArthur AG, McHugh D (1998) A biogeographical perspective of the deep-sea hydrothermal vent fauna. Adv Mar Biol 34:353-442

Underwood AJ, Petraitis P (1993) Structure of intertidal assemblages in different locations: How can local processes be compared? In: Ricklefs RE, Schluter D (eds) Species diversity in ecological communities. University of Chicago Press Chicago, p 39-51

Van Dover CL (2000) The ecology of deep-sea hydrothermal vents. Princeton University Press, Princeton, NJ

Van Dover $C L$, Desbruyères $D$, Segonzac $M$, Comtet $T$, Saldanha L, Fiala-Médioni A, Langmuir C (1996) Biology of the Lucky Strike hydrothermal vent field. Deep-Sea Res 43:1509-1529

Vanreusel A, Van den Bossche I, Thiermann F (1997) Freeliving marine nematodes from hydrothermal sediments: similarities with communities from diverse reduced habitats. Mar Ecol Prog Ser 157:207-219

Von Damm KL, Bray AM, Buttermore LG, Oosting SE (1998) The geochemical controls on vent fluids from the Lucky Strike vent field, Mid-Atlantic Ridge. Earth Planet Sci Lett 160.521-536

Wright DH, Currie DJ, Maurer BA (1993) Energy supply and patterns of species richness on local and regional scales. In: Ricklefs RE, Schluter D (eds) Species diversity in ecological communities. University of Chicago Press, Chicago, p $39-51,66-74$ 